\title{
Adaptive Backstepping Control of Uncertain Systems with Unknown Input Time-Delay
}

\author{
Jing Zhou* Wei Wang** Changyun Wen ${ }^{* *}$ \\ * Department of Engineering Cybernetics, Norwegian University of \\ Science and Technology, 7491 Trondheim, Norway \\ E-mail: jing.zhou@itk.ntnu.no \\ ** School of Electrical and Electronic Engineering, Nanyang \\ Technological University, Nanyang Avenue, 639798 Singapore \\ E-mail: ecywen@ntu.edu.sg, wang0336@ntu.edu.sg
}

\begin{abstract}
In this paper, we show that adaptive controllers designed using the standard backstepping technique proposed in Krstic [1995] globally stabilize a class of uncertain systems with unknown input time delay and unmodeled dynamics. As such systems belong to nonminimum phase systems and thus our result extends the class of systems stabilizable from minimum phase systems when the standard backstepping technique is employed. Moreover, it is shown that the transient system performance can be improved by adjusting the design parameters.
\end{abstract}

\section{INTRODUCTION}

Time-delay phenomenon is commonly found in chemical processes, biological reactors, rolling mills, communication networks, etc. Since the existence of delay usually deteriorates the performance of closed-loop system (Kolmanovskii and Nosov [1986], Malek-Zavarei and Jamshidi [1987]), the stabilization and control problem for time-delay systems have a topic of great importance and have received increasing attention. Some fruitful results have been achieved for past years when dealing with stabilizing problem for timedelay systems using backstepping technique. In Ge et al. [2003], neural network control cooperating with iterative backstepping was constructed for a class of nonlinear system with unknown but constant time delays. In Hua et al. [2005], time-varying delays were considered and the designed controller is independent of time delays. A decentralized feedback control approach for a class of large scale stochastic systems with time delay was proposed in $\mathrm{Wu}$ [2006]. More recently, in Hua et al. [2007] a result of backstepping adaptive tracking in the presence of time delay was established. All mentioned results are only applicable to systems with time delay in system states. However, when adopting backstepping approach, little attention has been focused on systems with time-delay in control input as such systems belong to non-minimum phase systems. Although Mazenc [2003] and Iasson [2006] did researches on such systems, the time delay and nonlinear system are all known and their approaches are non-adaptive state feedback control. Therefore, it is necessary and important to investigate adaptive backstepping of uncertain systems with unknown input time-delay.

In this paper, we present the standard backstepping design approach in Krstic [1995] without any modification to design adaptive controller for input time-delay systems in which delay is unknown. Such systems are also subject to unmodeled dynamics. It is shown that the designed controller can globally stabilize the system with a unknown delay of arbitrarily large length in the input asymptotically under certain conditions. We will use the technique in Wen et al. [1999] to deal with the unmodeled dynamics in the stability analysis.

The remaining part of the paper is organized as follows: Section 2 offers the class of systems to be controlled in this paper. Then adaptive control design scheme based on the backstepping approach is proposed in Section 3. After that, in Section 4 both of the robustness and transient performance analysises are established followed by an illustrative example presented to show the effectiveness of our proposed scheme in Section 5. Finally, the paper is concluded in Section 6.

\section{PROBLEM FORMULATION}

Consider the system with unknown input time delay and multiplicative unmodeled dynamics described as

$$
\begin{gathered}
y(t)=\frac{B(p)}{A(p)}\left(u(t)+\mu_{1} \Delta_{1}(p) u(t-\tau)\right) \\
+\mu_{2} \Delta_{2}(p) y(t)
\end{gathered}
$$

where $\tau$ is an unknown positive constant denoting time delay. $p$ denotes the differential operator $\frac{d}{d t}, B(p), A(p)$ and $\Delta_{1}(p), \Delta_{2}(p)$ are rational functions of $p$. With $p$ replaced by $s$, the corresponding $B(p), A(p)$ and $\Delta_{1}(p), \Delta_{2}(p)$ are transfer functions described as follows

$$
\begin{aligned}
& A(s)=s^{n}+a_{n-1} s^{n-1}+\ldots+a_{1} s+a_{0} \\
& B(s)=b_{m} s^{m}+\ldots+b_{1} s+b_{0} .
\end{aligned}
$$

$a_{i}(i=0,1, \ldots, n-1)$ and $b_{j}(j=0,1, \ldots, m)$ are unknown parameters, $\Delta_{1}(s)$ and $\Delta_{2}(s)$ are transfer functions of the unmodeled dynamics, $\mu_{1}$ and $\mu_{2}$ are positive scalars 
indicating the magnitudes of the unmodeled dynamics.

Our goal is to design a controller using backstepping technique to ensure the stability of the whole system and regulate the system output to zero. The system transient performance should also be adjustable by changing design parameters in certain sense.

For the system, we have the following assumptions.

Assumption 1: $B(s)$ is a Hurwitz polynomial. The order $n$, the sign of $b_{m}$ and the relative degree $\rho(=n-m)$ are known;

Assumption 2: $\Delta_{1}(s)$ and $\Delta_{2}(s)$ are stable, strictly proper and have a unity high frequency gain.

Obviously, system (1) has the following state space realization:

$$
\begin{aligned}
& \dot{x}=A x-a x_{1}+\left[\begin{array}{c}
0_{(\rho-1) \times 1} \\
b
\end{array}\right] u \\
& y=x_{1}+\mu_{1} \Delta_{1}(s) x_{1}(t-\tau)+\mu_{2} \Delta_{2}(s) y(t)
\end{aligned}
$$

where

$$
A=\left[\begin{array}{ccc}
0 & & \\
\vdots & I_{n-1} & \\
0 & \ldots & 0
\end{array}\right], a=\left[\begin{array}{c}
a_{n-1} \\
a_{n-2} \\
\vdots \\
a_{0}
\end{array}\right], b=\left[\begin{array}{c}
b_{m} \\
b_{m-1} \\
\vdots \\
b_{0}
\end{array}\right]
$$

In the design of adaptive controller, we only consider transfer function $\frac{B(s)}{A(s)}$, i.e,

$$
\begin{aligned}
& \dot{x}=A x-a x_{1}+\left[\begin{array}{c}
0_{(\rho-1) \times 1} \\
b
\end{array}\right] u, \\
& y=x_{1} .
\end{aligned}
$$

But in analysis, we will also take into account the effects of the time delay and the unmodelled dynamics, i.e.

$$
\mu_{1} \Delta_{1}(s) x_{1}(t-\tau)+\mu_{2} \Delta_{2}(s) y(t) .
$$

\section{DESIGN OF ADAPTIVE CONTROLLER}

In this section, we only present the adaptive controller designed using the standard backstepping technique in Krstic [1995], without giving the details. Firstly, a local filter using only local input and output is designed to estimate the states of system as follows:

$$
\begin{aligned}
& \dot{\eta}=A_{0} \eta+e_{n} y \\
& \dot{\lambda}=A_{0} \lambda+e_{n} u,
\end{aligned}
$$

where

$$
\begin{aligned}
A_{0} & =A-k e_{1}^{T}, \\
k & =\left[k_{1}, \ldots, k_{n}\right]^{T},
\end{aligned}
$$

the vector $k$ is chosen so that the matrix $A_{0}$ is Hurwitz, $e_{i}$ denotes the $i$ th coordinate vector in $\Re^{n}$. Then the state estimate of the system can be obtained by

$$
\hat{x}=-A_{0}^{n} \eta-\sum_{i=0}^{n-1} a_{i} A_{0}^{i} \eta+\sum_{i=0}^{m} b_{i} A_{0}^{i} \lambda .
$$

From system (4) and (8)-(12), the state estimation error $\epsilon=x-\hat{x}$ satisfies

$$
\dot{\epsilon}=A_{0} \epsilon+(a-k)\left(\mu_{1} \Delta_{1}(s) x_{1}(t-\tau)+\mu_{2} \Delta_{2}(s) y(t)\right)(13)
$$

Thus, the derivative of the system output can be expressed in the following form

$$
\begin{aligned}
\dot{y}= & x_{2}-a_{n-1} y+ \\
& \left(s+a_{n-1}\right)\left(\mu_{1} \Delta_{1}(s) x_{1}(t-\tau)+\mu_{2} \Delta_{2}(s) y(t)\right) \\
= & \xi_{2}+\omega^{T} \theta+\epsilon_{2}+ \\
& \left(s+a_{n-1}\right)\left(\mu_{1} \Delta_{1}(s) x_{1}(t-\tau)+\mu_{2} \Delta_{2}(s) y(t)\right),
\end{aligned}
$$

where

$$
\begin{aligned}
\theta & =\left[b^{T}, a^{T}\right] \\
\xi & =-A_{0}^{n} \eta \\
v_{j} & =A_{0}^{j} \lambda, \\
\omega & =\left[v_{m, 2}, v_{m-1,2}, \ldots, v_{0,2}, \Xi_{(2)}-y e_{1}^{T}\right]^{T} \\
\bar{\omega} & =\left[0, v_{m-1,2}, \ldots, v_{0,2}, \Xi_{(2)}-y e_{1}^{T}\right]^{T} \\
\Xi & =-\left[A_{0}^{n-1} \eta, \ldots, A_{0} \eta, \eta\right]
\end{aligned}
$$

and $v_{i, 2}, \epsilon_{2}, \xi_{2}, \Xi_{2}$ denote the second entries of $v_{i}, \epsilon, \xi, \Xi$ respectively.

The design system is now given as

$$
\begin{aligned}
\dot{y}= & \xi_{2}+\omega^{T} \theta+\epsilon_{2} \\
& +\left(s+a_{n-1}\right)\left(\mu_{1} \Delta_{1}(s) x_{1}(t-\tau)+\mu_{2} \Delta_{2}(s) y(t)\right) \\
\dot{v}_{m, i}= & v_{m, i+1}-k_{i} v_{m, 1}, \quad i=2, \ldots, \rho-1 \\
\dot{v}_{m, \rho}= & v_{m, \rho+1)}-k_{\rho} v_{m, 1}+u
\end{aligned}
$$

All states of the local filters in (8) and (9) are available for feedback. Even though the estimated state is given in (12), it is still unknown and thus can not be employed in the controller design. The state estimation error (13) and equation (21) will be utilized in system analysis.

Performing the standard backstepping procedures as in Krstic [1995], the controller designed for stabilizing system (1) can be summarized as follows where $\Gamma$ is a positive definite matrix in $\Re^{(n+m+1) \times(n+m+1)}, c_{i}, d_{i}$ and $\gamma$ are positive constants.

The change of coordinate is

$$
\begin{aligned}
& z_{1}=y \\
& z_{i}=v_{m, i}-\alpha_{i-1}, \quad i=2,3, \ldots, \rho,
\end{aligned}
$$

The control law is

$$
\begin{aligned}
u & =\alpha_{\rho}-v_{m, \rho+1} \\
\alpha_{1} & =\hat{\varrho} \bar{\alpha}_{1} \\
\bar{\alpha}_{1} & =-c_{1} z_{1}-d_{1} z_{1}-\xi_{2}-\bar{\omega}^{T} \hat{\theta} \\
\alpha_{2} & =-\hat{b}_{m} z_{1}-\left(c_{2}+d_{2}\left(\frac{\partial \alpha_{1}}{\partial y}\right)^{2}\right) z_{2}+\beta_{2}+\frac{\partial \alpha_{1}}{\partial \hat{\theta}} \Gamma \tau_{2} \\
\alpha_{i} & =-z_{i-1}-\left[c_{i}+d_{i}\left(\frac{\partial \alpha_{i-1}}{\partial y}\right)^{2}\right] z_{i}+\beta_{i}+\frac{\partial \alpha_{i-1}}{\partial \hat{\theta}} \Gamma \tau_{i}
\end{aligned}
$$




$$
\begin{aligned}
& -\sum_{k=2}^{i-1} \frac{\partial \alpha_{k-1}}{\partial \hat{\theta}} \Gamma \frac{\partial \alpha_{i-1}}{\partial y} z_{k},, i=3, \ldots, \rho \\
\beta_{i}= & \frac{\partial \alpha_{i-1}}{\partial y}\left(\xi_{2}+\omega^{T} \hat{\theta}\right)+k_{i} v_{m, 1}+\frac{\partial \alpha_{i-1}}{\partial \varrho} \hat{\varrho} \\
& +\frac{\partial \alpha_{i-1}}{\partial \eta}\left(A_{0} \eta+e_{n} y\right)+\sum_{j=1}^{m+i-1} \frac{\partial \alpha_{i-1}}{\partial \lambda_{j}}\left(-k_{j} \lambda_{1}\right. \\
& \left.+\lambda_{j+1}\right), i=3, \ldots, \rho
\end{aligned}
$$

with tuning functions

$$
\begin{aligned}
& \tau_{1}=\left(\omega-\varrho \hat{\varrho} \bar{\alpha}_{1} e_{1}\right) z_{1} \\
& \tau_{2}=\tau_{1}-\frac{\partial \alpha_{1}}{\partial y} \omega z_{2} \\
& \tau_{i}=\tau_{i-1}-\frac{\partial \alpha_{i-1}}{\partial y} \omega z_{i}
\end{aligned}
$$

The parameter updating laws are

$$
\begin{aligned}
& \dot{\hat{\varrho}}=-\gamma \operatorname{sgn}\left(b_{m}\right) \bar{\alpha}_{1} z_{1} \\
& \dot{\hat{\theta}}=\Gamma \tau_{\rho}
\end{aligned}
$$

Define $z(t)=\left[z_{1}, z_{2}, \ldots, z_{\rho}\right]^{T}$, with the above adaptive control law, the error system is characterized by

$$
\begin{aligned}
\dot{z}= & A_{z}(z, t) z+W_{\epsilon}(z, t) e_{2}^{T} \epsilon+W_{\theta}(z, t)^{T} \tilde{\theta}-b_{m} \bar{\alpha}_{1} e_{1} \tilde{\varrho} \\
& +W_{\epsilon}(z, t) . \\
& {\left[\left(s+a_{n-1}\right)\left(\mu_{1} \Delta_{1}(s) x_{1}(t-\tau)+\mu_{2} \Delta_{2}(s) y(t)\right)\right], }
\end{aligned}
$$

where

$$
\begin{aligned}
& A_{z}(z, t)=\left[\begin{array}{cc}
-c_{1}-d_{1} & \hat{b}_{m} \\
-\hat{b}_{m} & -c_{2}-d_{2}\left(\frac{\partial \alpha_{1}}{\partial y}\right)^{2} \\
0 & -1-\sigma_{2,3} \\
\vdots & \vdots \\
0 & -\sigma_{2, \rho}
\end{array}\right. \\
& \left.\begin{array}{ccc}
0 & \ldots & 0 \\
1+\sigma_{2,3} & \cdots & \sigma_{2, \rho} \\
-c_{3}-d_{3}\left(\frac{\partial \alpha_{2}}{\partial y}\right)^{2} & \ldots & \sigma_{3, \rho} \\
\vdots & \vdots & \vdots \\
-\sigma_{3, \rho} & \ldots & -c_{\rho}-d_{\rho}\left(\frac{\partial \alpha_{\rho-1}}{\partial y}\right)^{2}
\end{array}\right]
\end{aligned}
$$

the terms $\sigma_{i, j}$ are due to the terms $\frac{\partial \alpha_{i-1}}{\partial \hat{\theta}} \Gamma \frac{\partial \alpha_{j-1}}{\partial y} \omega$ in the $z_{j}$ equation.

\section{STABILITY ANALYSIS}

\subsection{Robustness Analysis}

Define Lyapunov function $V_{\rho}$ as follows:

$$
V_{\rho}=\sum_{i=1}^{\rho} \frac{1}{2} z_{i}^{2}+\frac{1}{2} \tilde{\theta}^{T} \Gamma^{-1} \tilde{\theta}+\frac{\left|b_{m}\right|}{2 \gamma} \tilde{\varrho}^{2}+\sum_{i=1}^{\rho} \frac{1}{d_{i}} \epsilon^{T} P \epsilon(40)
$$

where $P$ satisfies $P A_{0}+A_{0}^{T} P=-I, P=P^{T}>0$. From (13) and the designed controller (26)-(37), it can be shown that the derivative of $V_{\rho}$ satisfies

$$
\begin{aligned}
\dot{V}_{\rho} \leq & -\sum_{i=1}^{\rho_{i}} c_{i} z_{i}^{2}-\frac{d_{1}}{2}\left(z_{1}\right)^{2}+z_{1}\left(s+a_{n-1}\right)\left(\mu_{1} \Delta_{1}(s) .\right. \\
& \left.x_{1}(t-\tau)+\mu_{2} \Delta_{2}(s) y(t)\right)-\sum_{i=2}^{\rho}\left[\frac{d_{i}}{2}\left(\frac{\partial \alpha_{i-1}}{\partial y}\right)^{2} .\right. \\
& \left(z_{i}\right)^{2}-z_{i} \frac{\partial \alpha_{i-1}}{\partial y}\left(s+a_{n-1}\right)\left(\mu_{1} \Delta_{1}(s) x_{1}(t-\tau)\right. \\
& \left.\left.+\mu_{2} \Delta_{2}(s) y(t)\right)\right]-\sum_{i=1}^{\rho}\left[\frac{1}{2 d_{i}}\|\epsilon\|^{2}\right. \\
& \left.+\Phi^{T} \epsilon\left(\mu_{1} \Delta_{1}(s) x_{1}(t-\tau)+\mu_{2} \Delta_{2}(s) y(t)\right)\right] \\
\leq & -\sum_{i=1}^{\rho} c_{i}\left(z_{i}\right)^{2}-\sum_{i=1}^{\rho} \frac{1}{4 d_{i}}\|\epsilon\|^{2} \\
& +\sum_{i=1}^{\rho} \frac{1}{d_{i}} \mu_{1}^{2}\left\|\Delta_{1}(s)\left(s+a_{n-1)}\right) x_{1}(t-\tau)\right\|^{2} \\
& +\sum_{i=1}^{\rho} 2 d_{i}\|\Phi\|^{2}\left[\mu_{1}^{2}\left\|\Delta_{1}(s) x_{1}(t-\tau)\right\|^{2}\right. \\
& \left.+\mu_{2}^{2}\left\|\Delta_{2}(s) y\right\|^{2}\right] \\
& +\sum_{i=1}^{\rho} \frac{1}{d_{i}} \mu_{2}^{2}\left\|\Delta_{2}(s)\left(s+a_{n-1)}\right) y\right\|^{2}
\end{aligned}
$$

where

$$
\Phi^{T}=\sum_{i=1}^{\rho} \frac{2(a-k)^{T} P}{d_{i}}
$$

To show system stability, the variables of the filters in (8) and the zero dynamics should be included in the Lyapunov function. Under a similar transformation as in Krstic [1995], the variable $\zeta$ associated with the zero dynamics can be shown to satisfy

$$
\begin{aligned}
& \dot{\tilde{\zeta}}=A_{b} \tilde{\zeta}+b_{b} x_{1} \\
& \dot{\tilde{\eta}}=A_{0} \tilde{\eta}+e_{n} z_{1}
\end{aligned}
$$

where

$$
\begin{aligned}
\dot{\eta} & =A_{0} \eta+e_{n} z_{1} \\
\dot{\eta}^{r} & =A_{0} \eta^{r}, \tilde{\eta}=\eta-\eta^{r} \\
\dot{\zeta} & =A_{b} \zeta+b_{b} x_{1} \\
\dot{\zeta}^{r} & =A_{b} \zeta^{r}, \tilde{\zeta}=\zeta-\zeta^{r}
\end{aligned}
$$

where the eigenvalues of the matrix $A_{b} \in R^{m \times m}$ are the zeros of the Hurwitz polynomial $N(s)$ and $b_{b} \in R^{m}$.

$$
\begin{aligned}
& A_{b}=\left[\begin{array}{cccc}
-b_{m-1} / b_{m} & & & \\
& I_{m-1} & \\
\vdots & & & \\
-b_{0} / b_{m} & 0 & \ldots & 0
\end{array}\right] \text {, } \\
& b_{b}=T A^{\rho}\left[\begin{array}{l}
0 \\
b
\end{array}\right], T=\left[A_{b}^{\rho} e_{1}, \ldots, A_{b} e_{1}, I_{m}\right] .
\end{aligned}
$$

To deal with the unmodeled dynamics, we let $v_{1}$ and $v_{2}$ be the state vectors associated with $\Delta_{1}(s)$ and $\Delta_{2}(s)$ respectively. 


$$
\begin{aligned}
\dot{v}_{1} & =A_{1} v_{1}+b_{v 1} x_{1} \\
\Delta_{1}(s) x_{1} & =(1,0, \ldots, 0) v_{1} \\
\dot{v}_{2} & =A_{2} v_{2}+b_{v 2} z_{1} \\
\Delta_{2}(s) y & =\Delta_{2}(s) z_{1}=(1,0, \ldots, 0) v_{2}
\end{aligned}
$$

where $A_{1}$ and $A_{2}$ are stable from Assumption 2.

Lemma 1. The effects of the unmodeled dynamics are bounded as follows

$$
\begin{gathered}
\left\|\Delta_{1}(s) x_{1}(t-\tau)\right\|^{2} \leq\|\chi(t-\tau)\|^{2} \\
\left\|\Delta_{2}(s) y\right\|^{2} \leq\|\chi\|^{2}
\end{gathered}
$$

and

$$
\begin{aligned}
\left\|\Delta_{2}(s)\left(s+a_{n-1)}\right) y\right\|^{2} & \leq k_{3}\|\chi\|^{2} \\
\left\|\Delta_{1}(s)\left(s+a_{n-1)}\right) x_{1}(t-\tau)\right\|^{2} & \leq k_{1}\left\|x_{1}(t-\tau)\right\|^{2} \\
& +k_{2}\|\chi(t-\tau)\|^{2} \\
\left\|x_{1}(t)\right\|^{2} & \left.\leq\left(3+3 \mu_{2}^{2}\right)\right)\|\chi(t)\|^{2} \\
& +3 \mu_{1}^{2}\|\chi(t-\tau)\|^{2}
\end{aligned}
$$

where $\chi=\left[z^{T}, \epsilon^{T}, \tilde{\eta}^{T}, \xi, v_{1}^{T}, v_{2}^{T}\right]^{T}$.

Proof. (55) and (56) can be obtained directly from (52) and (54). Since $\Delta_{1}(s)$ and $\Delta_{2}(s)$ are strictly proper, we have

$$
\begin{aligned}
\left\|\Delta_{1}(s)\left(s+a_{n-1)}\right) x_{1}(t-\tau)\right\|^{2} & \leq k_{1}\left\|x_{1}(t-\tau)\right\|^{2} \\
& +k_{2}\|\chi(t-\tau)\|^{2} \\
\left\|\Delta_{2}(s)\left(s+a_{n-1)}\right) y\right\|^{2} & \leq k_{3}\|\chi\|^{2}
\end{aligned}
$$

From (4) and (6), it is known that

$$
x_{1}(t)=z_{1}(t)-\mu_{1} \Delta_{1}(s) x_{1}(t-\tau)-\mu_{2} \Delta_{2}(s) z_{1}(t)
$$

Thus

$$
\begin{aligned}
\left\|x_{1}(t)\right\|^{2} & =\left\|z_{1}(t)-\mu_{1} \Delta_{1}(s) x_{1}(t-\tau)-\mu_{2} \Delta_{2}(s) z_{1}(t)\right\|^{2} \\
& \left.\leq\left(3+3 \mu_{2}^{2}\right)\right)\|\chi(t)\|^{2}+3 \mu_{1}^{2}\|\chi(t-\tau)\|^{2}
\end{aligned}
$$

Theorem 1. Consider the closed-loop adaptive system consisting of the plant (1) under Assumptions 1 and 2, the controller (26), the parameter update laws (36), (37), and the filters (8) and (9). There exists a constant $\mu^{*}$ such that for all $\mu_{1}<\mu^{*}$ and $\mu_{2}<\mu^{*}$, all the signals in the system are globally uniformly bounded and $\lim _{t \rightarrow \infty}|y(t)|=0$ for arbitrary initial $x(0)$.

Proof. Introduce an augmented Lyapunov function as

$$
\begin{aligned}
\bar{V}= & V_{\rho}+\frac{1}{k_{\eta}} \tilde{\eta}^{T} P \tilde{\eta}+\frac{1}{k_{\zeta}} \tilde{\zeta}^{T} P_{b} \tilde{\zeta} \\
& +q_{1} v_{1}^{T} P_{1} v_{1}+q_{2} v_{2}^{T} P_{2} v_{2}
\end{aligned}
$$

where $P_{1}$ and $P_{2}$ satisfy $P_{1} A_{1}+A_{1}^{T} P_{1}=-I$ and $P_{2} A_{2}+$ $A_{2}^{T} P_{2}=-I$, respectively.

$$
\dot{\bar{V}}=\dot{V}_{\rho}-\frac{1}{k_{\eta}} \tilde{\eta}^{2}+\frac{2}{k_{\eta}} \tilde{\eta}^{T} P e_{n} z_{1}-\frac{1}{k_{\zeta}} \zeta^{2}+\frac{2}{k_{\zeta}} \tilde{\zeta}^{T} P_{b} b_{b} x_{1}
$$

$$
\begin{aligned}
& -q_{1}\left\|v_{1}\right\|^{2}+2 q_{1} v_{1}^{T} P_{1} b_{v 1} x_{1} \\
& -q_{2}\left\|v_{2}\right\|^{2}+2 q_{2} v_{2}^{T} P_{2} b_{v 2} z_{1} \\
& \leq-\frac{1}{2} c_{1} z_{1}^{2}-\sum_{i=2}^{\rho} c_{i} z_{i}^{2}-\sum_{i=1}^{\rho} \frac{1}{4 d_{i}}\|\epsilon\|^{2}-\frac{1}{2 k_{\eta}} \tilde{\eta}^{2} \\
& -\frac{1}{2 k_{\zeta}} \tilde{\zeta}^{2}-\frac{1}{2} q_{1}\left\|v_{1}\right\|^{2}-\frac{1}{2} q_{2}\left\|v_{2}\right\|^{2} \\
& +\sum_{i=1}^{\rho} \frac{1}{d_{i}} \mu_{1}^{2}\left\|\Delta_{1}(s)\left(s+a_{n-1)}\right) x_{1}(t-\tau)\right\|^{2} \\
& +\sum_{i=1}^{\rho} 2 d_{i}\|\Phi\|^{2} \mu_{1}^{2}\left\|\Delta_{1}(s) x_{1}(t-\tau)\right\|^{2} \\
& +\sum_{i=1}^{\rho} \frac{1}{d_{i}} \mu_{2}^{2}\left\|\Delta_{2}(s)\left(s+a_{n-1)}\right) y\right\|^{2} \\
& +\sum_{i=1}^{\rho} 2 d_{i}\|\Phi\|^{2} \mu_{2}^{2}\left\|\Delta_{2}(s) y\right\|^{2}-\frac{1}{4 k_{\zeta}} \tilde{\zeta}^{2} \\
& -\frac{2}{k_{\zeta}} \tilde{\zeta}^{T} P_{b} b_{b}\left(\mu_{1} \Delta_{1}(s) x_{1}(t-\tau)+\mu_{2} \Delta_{2}(s) z_{1}(t)\right) \\
& -\frac{1}{4} q_{1}\left\|v_{1}\right\|^{2}-2 q_{1} v_{1}^{T} P_{1} b_{v 1}\left(\mu_{1} \Delta_{1}(s) x_{1}(t-\tau)\right. \\
& \left.+\mu_{2} \Delta_{2}(s) z_{1}(t)\right) \\
& -\frac{1}{8} c_{1} z_{1}^{2}-\frac{1}{2 k_{\eta}} \tilde{\eta}^{2}+\frac{2}{k_{\eta}} \tilde{\eta}^{T} P e_{n} z_{1} \\
& -\frac{1}{8} c_{1} z_{1}^{2}-\frac{1}{4 k_{\zeta}} \tilde{\zeta}^{2}+\frac{2}{k_{\zeta}} \tilde{\zeta}^{T} P_{b} b_{b} z_{1} \\
& -\frac{1}{8} c_{1} z_{1}^{2}-\frac{1}{4} q_{1}\left\|v_{1}\right\|^{2}+2 q_{1} v_{1}^{T} P_{1} b_{v 1} z_{1} \\
& -\frac{1}{8} c_{1} z_{1}^{2}-\frac{1}{2} q_{2}\left\|v_{2}\right\|^{2}+2 q_{2} v_{2}^{T} P_{2} b_{v 2} z_{1}
\end{aligned}
$$

If $k_{\eta}, k_{\zeta}, q_{1}$ and $q_{2}$ are taken as

$$
\begin{aligned}
k_{\eta} & \geq \frac{16\left\|P e_{n}\right\|^{2}}{c_{1}} \\
k_{\zeta} & \geq \frac{32\left\|P_{b} b_{b}\right\|^{2}}{c_{1}} \\
q_{1} & \leq \frac{c_{1}}{32\left\|P_{1} b_{v 1}\right\|} \\
q_{2} & \leq \frac{c_{1}}{16\left\|P_{2} b_{v 2}\right\|}
\end{aligned}
$$

then

$$
\begin{aligned}
\dot{\bar{V}} \leq & -\beta\|\chi\|^{2}+\left(\sum_{i=1}^{\rho} \frac{1}{d_{i}} k_{3}+\sum_{i=1}^{\rho} 2 d_{i}\|\Phi\|^{2}\right. \\
& \left.+\frac{8}{k_{\zeta}}\left\|P_{b} b_{b}\right\|^{2}+8 q_{1}\left\|P_{1} b_{v 1}\right\|^{2}\right) \mu_{2}^{2}\|\chi\|^{2} \\
& +\left(\sum_{i=1}^{\rho} \frac{1}{d_{i}} k_{2}+\sum_{i=1}^{\rho} 2 d_{i}\|\Phi\|^{2}+\frac{8}{k_{\zeta}}\left\|P_{b} b_{b}\right\|^{2}\right. \\
& \left.+8 q_{1}\left\|P_{1} b_{v 1}\right\|^{2}\right) \mu_{1}^{2}\|\chi(t-\tau)\|^{2} \\
& +\sum_{i=1}^{\rho} \frac{1}{d_{i}} k_{1} \mu_{1}^{2}\left\|x_{1}(t-\tau)\right\|^{2}-\frac{1}{4} c_{1} z_{1}^{2}
\end{aligned}
$$




$$
\begin{aligned}
= & -\frac{1}{4} c_{1} z_{1}^{2}-\beta\|\chi\|^{2}+\left(k_{3} k_{4}+k_{5}\right) \mu_{2}^{2}\|\chi\|^{2} \\
& +\left(k_{2} k_{4}+k_{5}\right) \mu_{1}^{2}\|\chi(t-\tau)\|^{2} \\
& +k_{1} k_{4} \mu_{1}^{2}\left\|x_{1}(t-\tau)\right\|^{2}
\end{aligned}
$$

where

$$
\begin{aligned}
\beta & =\min \left\{\frac{c_{1}}{4}, c_{2}, \ldots, c_{\rho}, \sum_{i=1}^{\rho} \frac{1}{4 d_{i}}, \frac{1}{2 k_{\eta}}, \frac{1}{2 k_{\zeta}}, \frac{1}{2} q_{1},\right. \\
k_{4} & \left.=\sum_{i=1}^{\rho} \frac{1}{2} q_{2}\right\} \\
k_{5} & =\sum_{i=1}^{\rho} 2 d_{i}\|\Phi\|^{2}+\frac{8}{k_{\zeta}}\left\|P_{b} b_{b}\right\|^{2}+8 q_{1}\left\|P_{1} b_{v 1}\right\|^{2}(73
\end{aligned}
$$

To tackle the unknown time-delay problem, we introduce the following Lyapunov-Krasovskii function

$$
\begin{aligned}
W= & k_{1} k_{4} \mu_{1}^{2} \int_{t-\tau}^{t}\left\|x_{1}(s)\right\|^{2} d s \\
& +\int_{t-\tau}^{t}\left(\left(k_{2} k_{4}+k_{5}\right) \mu_{1}^{2}+3 k_{1} k_{4} \mu_{1}^{4}\right)\|\chi(s)\|^{2} d
\end{aligned}
$$

Then the final Lyapunov function is

$$
V=\bar{V}+W
$$

The time derivative of $V$ is

$$
\begin{aligned}
\dot{V} \leq & -\beta\|\chi\|^{2}+\left(k_{3} k_{4}+k_{5}\right) \mu_{2}^{2}\|\chi\|^{2}-\frac{1}{4} c_{1} z_{1}^{2} \\
+ & \left(k_{2} k_{4}+k_{5}\right) \mu_{1}^{2}\|\chi(t-\tau)\|^{2}+k_{1} k_{4} \mu_{1}^{2}\left\|x_{1}(t-\tau)\right\|^{2} \\
& +k_{1} k_{4} \mu_{1}^{2}\left\|x_{1}(t)\right\|^{2}-k_{1} k_{4} \mu_{1}^{2}\left\|x_{1}(t-\tau)\right\|^{2} \\
& +\left(\left(k_{2} k_{4}+k_{5}\right) \mu_{1}^{2}+3 k_{1} k_{4} \mu_{1}^{4}\right)\|\chi\|^{2} \\
& -\left(\left(k_{2} k_{4}+k_{5}\right) \mu_{1}^{2}+3 k_{1} k_{4} \mu_{1}^{4}\right)\|\chi(t-\tau)\|^{2} \\
= & -\beta\|\chi\|^{2}+\left(k_{2} k_{4}+k_{5}\right) \mu_{1}^{2}\|\chi(t-\tau)\|^{2} \\
& +\left(\left(k_{3} k_{4}+k_{5}\right) \mu_{2}^{2}+k_{1} k_{4} \mu_{1}^{2}\left(3+3 \mu_{2}^{2}\right)\right)\|\chi(t)\|^{2} \\
& +3 k_{1} k_{4} \mu_{1}^{4}\|\chi(t-\tau)\|^{2}+\left(\left(k_{2} k_{4}+k_{5}\right) \mu_{1}^{2}\right. \\
& \left.+3 k_{1} k_{4} \mu_{1}^{4}\right)\|\chi\|^{2}-\frac{1}{4} c_{1} z_{1}^{2} \\
& -\left(\left(k_{2} k_{4}+k_{5}\right) \mu_{1}^{2}+3 k_{1} k_{4} \mu_{1}^{4}\right)\|\chi(t-\tau)\|^{2} \\
= & -\left[\beta-\left(\left(k_{3} k_{4}+k_{5}\right) \mu_{2}^{2}+k_{1} k_{4} \mu_{1}^{2}\left(3+3 \mu_{2}^{2}\right)\right)\right. \\
& \left.-\left(\left(k_{2} k_{4}+k_{5}\right) \mu_{1}^{2}+3 k_{1} k_{4} \mu_{1}^{4}\right)\right]\|\chi\|^{2}-\frac{1}{4} c_{1} z_{1}^{2} \\
\leq & -\frac{1}{4} c_{1} z_{1}^{2}-\left(\beta-\left(3 k_{1} k_{4}+k_{2} k_{4}+k_{3} k_{4}+2 k_{5}\right) \mu^{2}\right. \\
& \left.-6 k_{1} k_{4} \mu^{4}\right)\|\chi\|^{2}
\end{aligned}
$$

where
By taking $\mu^{*}$ as

$$
\mu=\max \left\{\mu_{1}, \mu_{2}\right\}
$$

$$
\mu^{*}=\sqrt{\frac{\sqrt{K_{1}^{2}+2 K_{2} \beta}+K_{1}}{K_{2}}},
$$

where,

$$
\begin{aligned}
& K_{1}=3 k_{1} k_{4}+k_{2} k_{4}+k_{3} k_{4}+2 k_{5}^{2} \\
& K_{2}=12 k_{1} k_{4}
\end{aligned}
$$

we have

$$
\dot{V} \leq-\frac{1}{4} c_{1} z_{1}^{2} \leq 0 .
$$

This concludes that all the signals in the system are globally uniformly bounded, and $\lim _{t \rightarrow \infty}|y(t)|=0$ for arbitrary initial $x(0)$.

Remark 1. This theorem shows that the designed controller is able to stabilize the system with unknown input delay and unmodeled dynamics whose magnitudes $\mu_{1}$ and $\mu_{2}$ are bounded by parameter $\mu^{*}$ satisfying (78). By choosing appropriate Lyapunov-Krasovskii function, the effect of input time delay can be compensated.

\subsection{Transient performance}

The bounds for system output $y(t)$ on both $L_{2}$ and $L_{\infty}$ norms can be stated in the following theorem.

Theorem 2. Consider the initial values $z_{i}(0), i=2, \ldots, \rho$, $\tilde{\eta}(0)=0, \tilde{\zeta}(0)=0, v_{1}(0)=0$ and $v_{2}(0)=0$, the $L_{2}$ and $L_{\infty}$ norms of output $y(t)$ are given by

$$
\begin{aligned}
\|y(t)\|_{2} \leq \frac{2}{\sqrt{c_{1}}} & {\left[\frac{1}{2} y(0)^{2}+\frac{1}{2}\|\tilde{\theta}(0)\|_{\Gamma^{-1}}^{2}\right.} \\
& \left.+k_{4}\|\epsilon(0)\|_{P}^{2}+\frac{\left|b_{m}\right|}{2 \gamma}|\tilde{\varrho}(0)|^{2}\right]^{1 / 2} \\
\|y(t)\|_{\infty} \leq \sqrt{2} & {\left[\frac{1}{2} y(0)^{2}+\frac{1}{2}\|\tilde{\theta}(0)\|_{\Gamma^{-1}}^{2}\right.} \\
+ & \left.k_{4}\|\epsilon(0)\|_{P}^{2}+\frac{\left|b_{m}\right|}{2 \gamma}|\tilde{\varrho}(0)|^{2}\right]^{1 / 2}
\end{aligned}
$$

where

$$
\begin{aligned}
\|\tilde{\theta}(0)\|_{\Gamma^{-1}}^{2} & =\tilde{\theta}^{T}(0) \Gamma^{-1} \tilde{\theta}(0) \\
\|\epsilon(0)\|_{P}^{2} & =\epsilon^{T}(0) P \epsilon(0)
\end{aligned}
$$

Proof. As shown in (81), $V$ is non-increasing, thus we have

$$
\begin{aligned}
\|y(t)\|_{2}^{2}=\int_{0}^{\infty}\left\|z_{1} t\right\|^{2} d t & \leq \frac{4}{c_{1}}(V(0)-V(\infty)) \\
& \leq \frac{4}{c_{1}}(V(0)) \\
\|y(t)\|_{\infty} & \leq \sqrt{2 V(0)}
\end{aligned}
$$

From (46) and (48), we can set $\tilde{\eta}(0)=0$ and $\tilde{\zeta}(0)=0$ by choosing $\eta^{r}(0)=\eta(0)$ and $\zeta^{r}(0)=\zeta(0)$. Consider the zero initial values 


$$
\tilde{\eta}(0)=0, \tilde{\zeta}(0)=0, v_{1}(0)=0, v_{2}(0)=0
$$

Besides, from (25), the initial values $z_{i}(0), i=2, \ldots, \rho$ can be set to zero by suitably choosing $v_{m, i}$ as

$$
\begin{gathered}
v_{m, i}(0)=\alpha_{i-1}\left(y(0), \hat{\theta}(0), \hat{\varrho}(0), \eta(0), \lambda(0), v_{m, i-1}(0)\right), \\
i=2, \ldots, \rho
\end{gathered}
$$

Thus we have

$$
\begin{aligned}
V(0)=\frac{1}{2} y(0)^{2}+\frac{1}{2}\|\tilde{\theta}(0)\|_{\Gamma^{-1}}^{2} & +k_{4}\|\epsilon(0)\|_{P}^{2} \\
& +\frac{\left|b_{m}\right|}{2 \gamma}|\tilde{\varrho}(0)|^{2}
\end{aligned}
$$

Substituting the above results to (86) and (87), we will get (82) and (83) respectively.

Remark 2. This theorem shows that the $L_{2}$ norm and $L_{\infty}$ norm of output $y$ depend on the initial estimation errors $\tilde{\theta}(0), \tilde{\epsilon}(0)$ and $\tilde{\varrho}(0)$. The closer the initial estimates to the true values, the better the transient performance turns out. The bounds can be reduced by increasing $c_{1}$ (82), $\Gamma$ and $\gamma(82,83)$.

\section{AN ILLUSTRATIVE EXAMPLE}

We illustrate the approach on a relative-degree-two timedelay system with unmodeled dynamics as described in (1), where $B(s)=b_{1} s+b_{0}=2 s+3$ and $A(s)=s^{3}+$ $a_{2} s^{2}+a_{1} s+a_{0}=s^{3}+3 s^{2}+2 s$, delay $\tau=2$ seconds. Note that $a_{2}, a_{1}, a_{0}, b_{1}, b_{0}$ and $\tau$ are all considered to be unknown in controller design. Meanwhile, the order $n=3$, the sign of $b_{1}$ and the relative degree $\rho=2$ are known. The unmodeled dynamics $\Delta_{1}=\Delta_{2}=\frac{1}{s+1}$ and the constants $\mu_{1}=\mu_{2}=0.1$. In simulation, all the initials are set as 0 except for $y(0)=0.4$ and $\varrho(0)=0.2$.

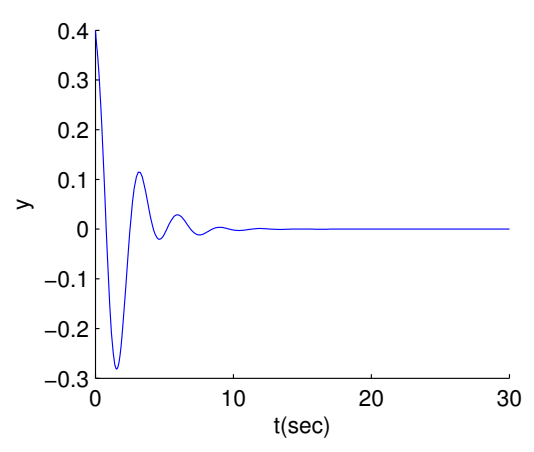

Fig. 1. The output $y$ of time-delay system with unmodeled dynamics

The design parameters are chosen as vector $k=[6,12,8]^{T}$, $c_{1}=c_{2}=2$ and $d_{1}=d_{2}=0.5$. With the the presented adaptation mechanism on by choosing $\gamma=0.1$ and $\Gamma=0.1 \times I$, the results are sketched in Fig.(1)-(2), which obviously show that the system can be stablized and the system output converge to zero even certain unknown time delay in the input and unmodeled dynamics exist.

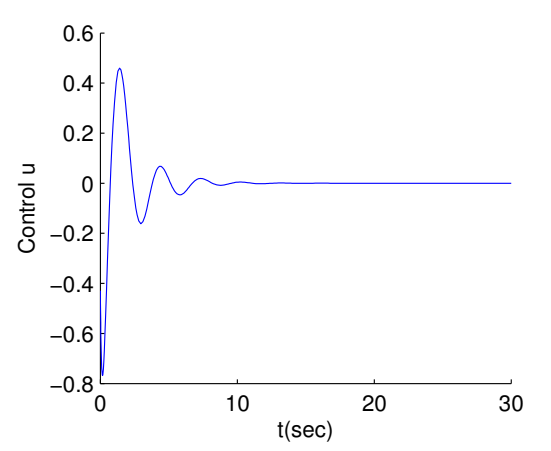

Fig. 2. Control input $u$

\section{CONCLUSION}

Adaptive controllers using the standard backstepping technique without modification are shown robust with respect to time delay in system input and unmodelled dynamics. This implies that the class of systems stabilizable using backstepping adaptive controllers can be extended to certain non-minimum phase systems. Transient system performance can also be improved by adjusting the design parameters.

\section{REFERENCES}

V. B. Kolmanovskii and V. R. Nosov. Stability of Functional Differential Equations. New York: Academic Press, 1986.

M. Malek-Zavarei and M. Jamshidi. Time-delay systems : analysis, optimization, and applications. Amsterdam: North-Holland, 1987.

M. Krstic, I. Kanellakopoulos, and P. V. Kokotovic. Nonlinear and Adaptive Control Design. New York: Wiley, 1995.

C. Wen, Y. Zhang, and Y. C. Soh. Robustness of an adaptive backstepping controller without modification Systems and Control Letters, vol. 36, pages 87-100, 1999.

S. S. Ge, F. Hang, and T. H. Lee. Adaptive Neural Network Control of Nonlinear Systems With Unknown Time Delays IEEE Transactions on Automatic Control, vol. 48, pages 4524-4529, 2003

C. Hua, X. Guan, and P. Shi. Robust Output Feedback Tracking Control for Time-Delay Nonlinear Systems Using Neural Network IEEE Transactions on Neural Networks, vol. 18, pages 495-505, 2007.

K. Iasson. Finite-Time Global Stabilization by Means of Time-Varying Distributed Delay Feedback SIAM Journal on Control and Optimization, vol. 45, pages 320-342, 2006.

S. Wu, F. Deng and S. Xiang. Backstepping controller design for large-scale stochastic systems with time delays Proceedings of the World Congress on Intelligent Control and Automation (WCICA), pages 1181-1185, 2006.

F. Mazenc and P. A. Bliman. Backstepping Design for Time-Delay Nonlinear Systems 42nd IEEE International Conference on Decision and Control, pages 45514556, 2003.

C. Hua, X. Guan, and P. Shi. Robust backstepping control for a class of time delayed systems IEEE Transactions on Automatic Control, vol. 50, pages 894-899, 2005. 